\title{
Algeria's preparedness for Omicron variant and for the fourth wave of COVID-19
}

\author{
Hani Amir Aouissi* \\ Scientific and Technical Research Center on Arid Regions (CRSTRA), Biskra, Algeria.
}

\begin{abstract}
The world has been confronting a health crisis for two years because of the COVID-19 pandemic. The context of the crisis has the form of the first wave and subsequent waves that varied depending on the country. Undoubtedly, Algeria is one of the countries that have suffered severely from this pandemic. The recent wave has been marked with the huge number of casualties given the poor preparation. The country's preparation issue will be more complicated. In other words, in the context of an expected 4th wave that is characterized with the appearance of the new Omicron variant B.1.1.529. Thus, this news will consider the situation comprehensively and will provide recommendations to minimize the potential damage that will result from the fourth wave, and to attempt to limit the spread of the virus. The updated measures aim at rising the opportunities of improving the health situation in the country in the near future.
\end{abstract}

Keywords: COVID-19, pandemic, Algeria, Omicron, global health

Similar to many countries, Algeria has extensively suffered from COVID-19. The first case was reported on February 25, 2020. Early 2021, Algeria has shocked the world by reporting one of the highest fatality rate with approximately $15 \%$ (1).

So many countries announced officially the fourth wave of COVID-19. In most cases, this wave was quite deadly despite the democratization of vaccination. Concerning Algeria, many specialists predicted a next 4th wave around December 2021. In this regard, a lot of works has recently been published concerning predictive models of cases, deaths or future waves of the virus in the country (2). However, in most cases the spread of this virus has often differed from the predictions.

Despite governmental efforts, there are still deficiencies in testing; meaning the number of infections is likely to be an underestimate of actual numbers because they are based on PCRs only.

Thus, the question that arises is: "Is Algeria ready for a 4th wave?"

Firstly, throughout the 3rd wave, the country experienced a rather murderous period by recording 1927 cases and 49 deaths the July 28, 2021 (Figure 1). At that period, the country was already poorly prepared. This is in particular with the appearance of the Delta variant, which caused a lot of damage and led to the emergence of several clusters. The damage was obvious especially in the northern cities as they represent the most densely populated areas. Therefore, the third wave has caused many deaths that could have been avoided specifically in people suffering from comorbidities (3).

Further, despite the fact that Algeria has ordered fairly large doses of vaccines (AstraZeneca, Sputnik $\mathrm{V}$, and Sinovac), the vaccination remains quite humble because only $11 \%$ of the population is fully vaccinated (4). Some surveys have revealed that people do not have a firm confidence in vaccination (5), which might justify the vaccine refusal from a large majority of the population.

Fundamentally, facts like the growing lack of severity in the application of preventive measures, the gradual opening of borders will most certainly be unhelpful in dealing with the issue.

In addition to the previous reasons, the appearance of the Omicron variant in South Africa, which is already present in 57 countries according to the World Health Organization (WHO) (6), will make the situation firmer and more delicate to treat. The variant was characterized by an impressive diffusion speed, particularly in the neighbouring countries of South Africa (Eswatini, Zimbabwe, Mozambique, Namibia and Lesotho). While currently little information is provided, scientists predict that after the third dose, the variant is unlikely to be more dangerous than the Delta variant which remains dominant. Actually, there has yet been no detection in Algeria, but everything suggests that it is only a matter of time.

In conclusion, to avoid a fourth wave's significant 


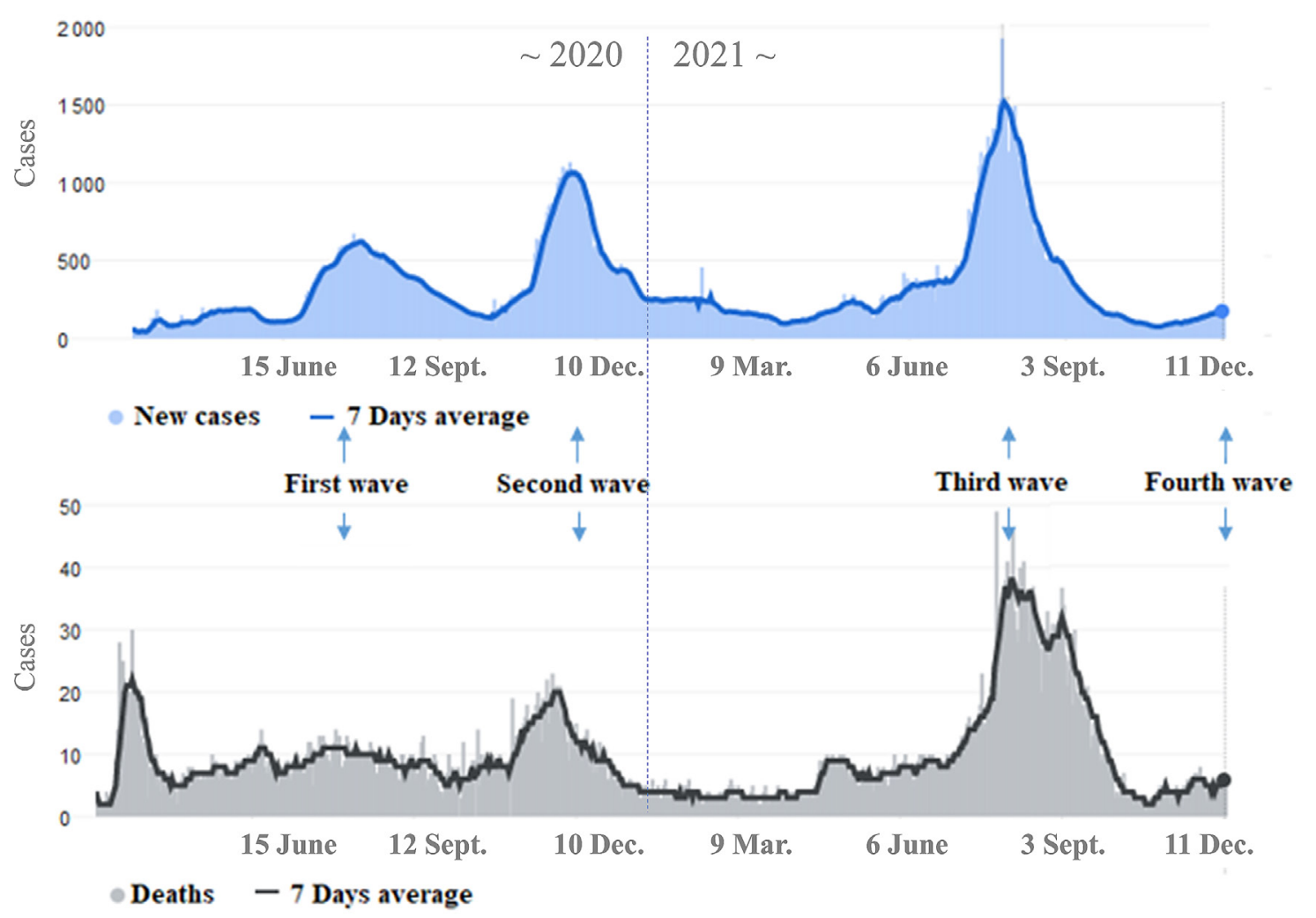

Figure 1. Daily cases and deaths during the three waves of COVID-19 pandemic in Algeria. (Data source: $h t t p s: / /$ coronavirus.jhu.edu/map.html).

losses, the continuity of the strict application of protective measures (wearing a mask, washing hands, social distancing, etc.) and making the necessary arrangements for the availability of oxygen are recommended. It is also necessary to perform an intensive vaccination process to optimise protection.

\section{Funding: None.}

Conflict of Interest: The author has no conflict of interest to disclose.

\section{References}

1. Ababsa M, Aouissi HA. Current state of the coronavirus (Covid-19) in Algeria. J Community Med Health Care. 2020; 5: 1036.

2. Lounis M, Torrealba-Rodriguez O, Conde-Gutiérrez RA. Predictive models for COVID-19 cases, deaths and recoveries in Algeria. Results Phys. 2021; 104845.

3. Aouissi HA, Belhaouchet I. What about rheumatic diseases and COVID-19? New Microbes New Infect. 2021; 41:100846.
4. Mathieu E, Ritchie H, Ortiz-Ospina E, Roser M, Hasell J, Appel C, Giattino C, Rodés-Guirao L. A global database of COVID-19 vaccinations. Nat Hum Behav. 2021; 5:947-953.

5. Qunaibi E, Basheti I, Soudy M, Sultan I. Hesitancy of Arab Healthcare Workers towards COVID-19 Vaccination: A Large-Scale Multinational Study. Vaccines (Basel). 2021; 9:446.

6. Global NEWS. Omicron COVID-19 variant has spread to 57 countries, number expected to increase: WHO. https:// globalnews.ca/video/8433680/omicron-covid-19-varianthas-spread-to-57-countries-number-expected-to-increasewho (accessed December 8, 2021).

Received November 19, 2021; Revised December 12, 2021; Accepted December 14, 2021.

Released online in J-STAGE as advance publication December 16, 2021.

\section{*Address correspondence to:}

Hani Amir Aouissi, Scientific and Technical Research Centre on Arid Regions (CRSTRA), Campus Mohamed Kheider, BP 1682, El Alia, Biskra 07000, Algeria.

E-mail: aouissi.amir@gmail.com / hani.amir.aouissi@crstra.dz 